\title{
Direct optical observation of walls and disclination effects in active photonic devices
}

\author{
B. D. Snow ${ }^{1 \dagger}$, F. R. M. Adikan ${ }^{1}$, J. C. Gates ${ }^{1}$, C. B. E. Gawith ${ }^{1}$, \\ A. Dyadyusha ${ }^{2}$, Huw E. Major ${ }^{1}$, M. Kaczmarek ${ }^{2}$, and P. G. R. Smith ${ }^{1}$ \\ ${ }^{1}$ Optoelectronics Research Centre, University of Southampton, \\ Southampton, SO17 1BJ, United Kingdom \\ ${ }^{2}$ School of Physics and Astronomy, University of Southampton, \\ Southampton, SO17 1BJ, United Kingdom \\ †bds@orc.soton.ac.uk
}

\begin{abstract}
Liquid crystal tunable Bragg Gratings defined in planar substrates via a laser patterning technique exhibit complex wavelength tuning. This tuning displays threshold points and hysteresis. These tuning features are shown to be a manifestation of physical processes occurring in the confined geometry of our tunable devices. Such physical processes include the formation and removal of line disclinations and an associated wall. We discuss the effect of walls in the liquid crystal with regards to voltage tuning characteristics and whether they may allow faster wavelength tuning.
\end{abstract}

Keywords: liquid crystals, optics, Bragg gratings

\section{Introduction}

Liquid crystals (LC) are increasingly finding uses in fields outside of display optics. Their strong electro-optic response can be used in applications such as tunable photonic devices, including tunable planar Bragg gratings. While Bragg gratings are well known as fixed wavelength reflectors the application of a liquid crystal can convert these fixed reflectors into tunable filter elements, with potential applications in telecommunications networks [1].

In our previous work on planar lightwave circuits, we have demonstrated the capability to tune the Bragg peak through adjacent DWDM channels [2]. These devices contained channel waveguides and gratings defined by laser writing techniques. The Bragg peak is tuned by applying an electric field across the device, which causes a change in the LC refractive index, and in-turn modifies the modal index of an exposed Bragg grating. Surprisingly, the tuning curves for these devices exhibit hysteresis depending on whether the field amplitude is being increased or decreased. In this work we report investigation of the liquid crystal behaviour by direct observation of polarization state and physical changes in the LC.

\section{Background}

In our previous work we determined the electrical tunability range of planar Bragg gratings with a liquid crystal overlayer. Our direct-UV-written silica-on-silicon tunable LC devices exhibited a Bragg wavelength shift of $932.7 \mathrm{pm}$ (corresponding to $114 \mathrm{GHz}$ at $\lambda=1561.8 \mathrm{~nm}$ for TE polarization) with $170 \mathrm{Vpp}$ applied across the LC cell $[2,3]$. 


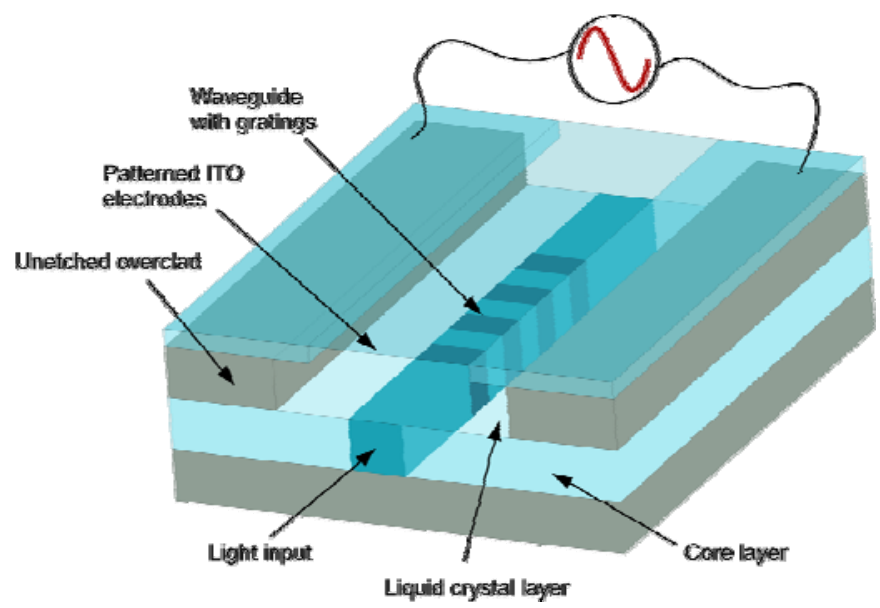

Figure 1: Schematic illustrating a liquid crystal tunable planar Bragg grating device

A schematic illustrating the main features of the tunable devices fabricated in this work is shown in Figure 1. Our previous work showed that the tuning behaviour of these devices is dependent upon the history of the LC reorientation, which is governed by the applied voltage. As such, the increasing and decreasing voltage regimes displayed different behaviour. This differing response is seen as hysteresis in the tuning curves, such that the Bragg wavelength peak tuning is not a linear function of applied voltage. The voltage versus Bragg centre wavelength tuning curve of our devices is shown in Figure 2.

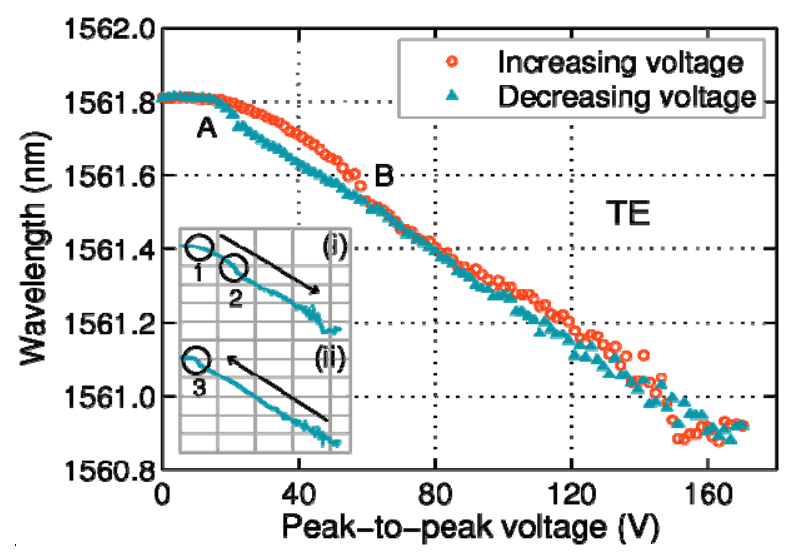

Figure 2: A graph showing the electrically-induced tuning response of a liquid crystal planar Bragg grating device exhibiting hysteresis

In the graph of Figure 2 a deviation from a linear response is seen between points $\mathrm{A}$ and B. Here, the tuning curves for increasing voltage in inset (i) and the decreasing voltage of inset (ii) differ, resulting in hysteresis. Voltages where the gradient of the tuning curve varies considerably are circled and labelled as 1,2 and 3 . The observations that will be presented in this paper suggest that LC molecular reorientation differs for the increasing and decreasing voltage regimes. Below point $\mathrm{A}$, the applied field strength is insufficient to cause the Freedericksz transition. However, above point A the applied field 
is sufficient to overcome the effect of surface pinning and the equilibrium condition is no longer uniform. Above point $\mathrm{B}$, the tuning is linear for both increasing and decreasing voltage regimes.

Our previous laser-written LC tunable devices were fabricated in silica-on-silicon substrates (Figure 1). To analyze the LC alignment processes in-situ we fabricated physically and electrically equivalent structures to our original devices, but which were optically transparent to allow direct observation. A straight $\sim 50 \mu \mathrm{m}$ wide gap was scribed into an ITO coated coverslip to form two parallel electrodes. The ITO was coated with surfactant for homeotropic alignment and Merck nematic LC 18523 was applied to the cell. Transmission microscopy experiments were performed through crossed polarizers and the resultant polarization changes inside the LC cell recorded.

\section{Results and discussion}

Our observations suggest that the hysteretic behaviour seen in our previous work [4] can be linked to the domain wall and disclination dynamics of the LC. The shape of the domain wall is largely governed by the geometry of the electrodes and less so by surface defects. As the wall and subsequent disclinations form above our waveguides, any change in the refractive index of the LC due to director realignment will manifest itself as a change in centre wavelength. The transparent LC cells showed a wall forming between the electrodes that narrowed and subsequently formed two disclinations at higher voltages through the pincement transition.

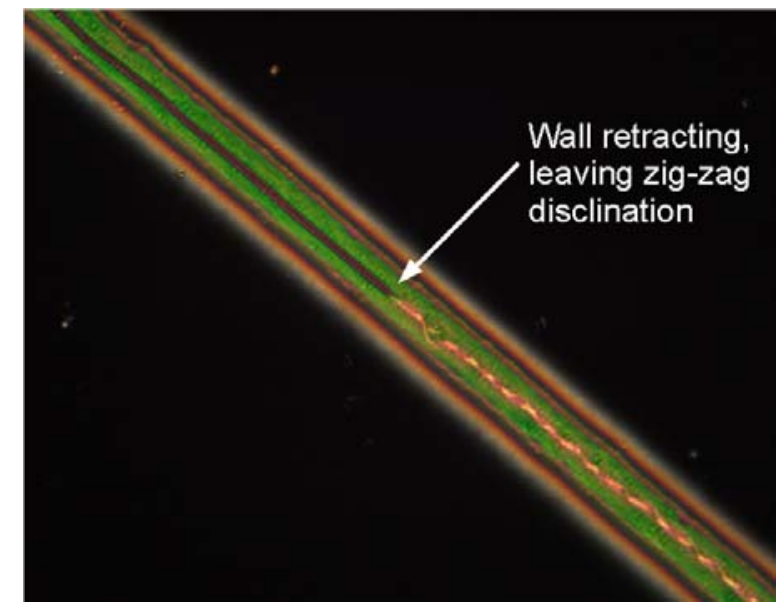

Figure 3: Wall dynamics seen via crossed polarizers when ramping an a.c. voltage up.

Figure 3 shows that the wall retreats at higher fields when the LC becomes fullyaligned with the applied field. Figure 4 shows the two zig-zag disclinations formed as the distortion in the wall becomes large and the wall transforms into two lines. Here, a defect is added on the lower surface to separate the two disclinations and the cell is illuminated with narrowband light centered at $516 \mathrm{~nm}$. The top disclination is in focus and is situated in a plane above the defect. The critical voltage values from the transparent cells agree with tuning curve threshold points. 


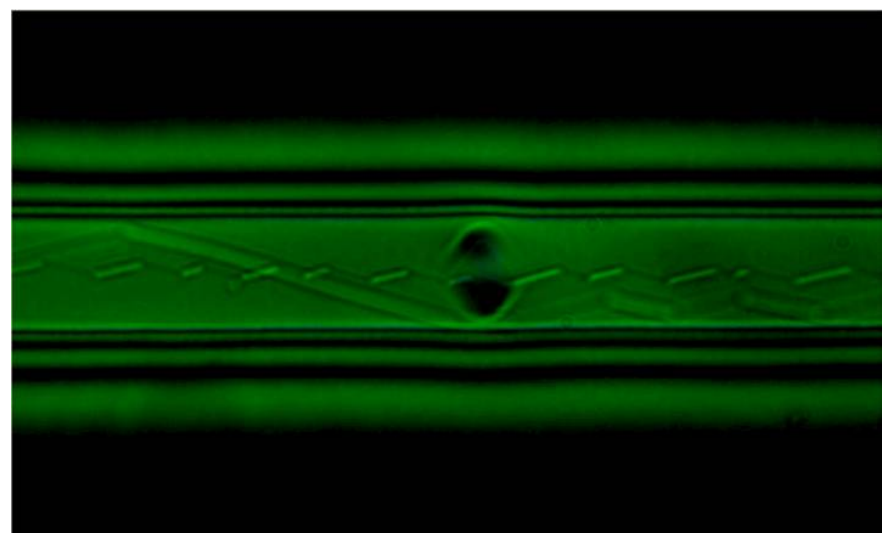

Figure 4: Zig-zag disclinations formed from the pincement transition

The tuning previously noted exhibits hysteresis but is linear at higher voltages. It can therefore be assumed that the ratio of area bounded by the wall to regions of field aligned director varies with voltage. Indeed, in our previous work point defects have been seen along the wall [2]. These form nucleation points from which the wall can retreat. Thus the percentage of the waveguide covered by the wall decreases with increasing voltage, and so the change in the effective refractive index of the propagating optical mode is not necessarily a linear function of applied voltage. The appearance and disappearance of the wall structure is shown in the photographs of Figure 5. Figures 5 (ad) show the increasing voltage regime, and (e-h) show the decreasing voltage regime.

In our current work we aim to investigate whether wall dynamics can be controlled by the application of periodic defects along the electrode region. This potentially allows for faster wall retraction, aiding tuning speed and response characteristics of the tuning devices.

\section{Conclusions}

We have previously demonstrated electro-optical tuning of a planar Bragg grating device using a LC overlay. The tuning curves were shown to exhibit hysteresis which is the result of wall dynamics in the electrode region of our devices. We have presented new results on high resolution optical observation of wall dynamics. This data indicates physical mechanisms are responsible for the features seen in our voltage tuning curves. Most notably the key voltage points have been associated with the formation and removal of the wall structure. These results, together with similar measurements in vertical cell arrangements, support the relationship between wall formation and the observed hysteresis in spectral tuning. 


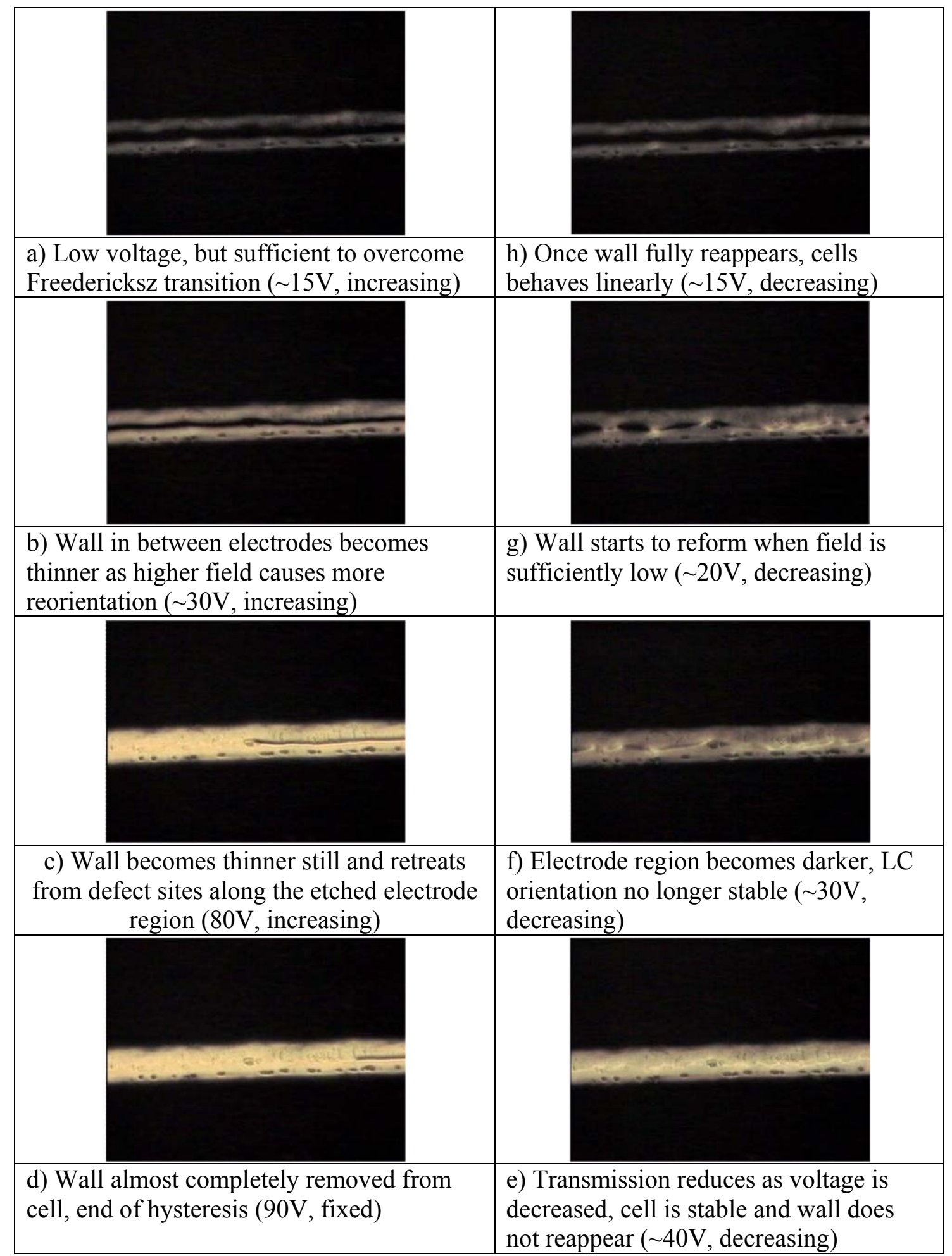

Figure 5: Photographs of wall dynamics in a homeotropically aligned cell when ramping an a.c. voltage up (a-d) and down (e-h) 


\section{References}

[1] L. Sirleto, L. Petti, P. Mormille, G.C. Righini, G. Abbate. Fiber Integr. Opt., 21, 435, (2002).

[2] F. R. M. Adikan, J. C. Gates, A. Dyadyusha, H. E. Major, C. B. E. Gawith, I. J. G. Sparrow, G. D. Emmerson, M. Kaczmarek, P. G. R. Smith. Opt. Lett., 32, 1542, (2007).

[3] G. D. Emmerson, S. P. Watts, C. B. E. Gawith, V. Albanis, M. Ibsen, R. B. Williams, P. G. R. Smith. Elec. Lett., 38, 1531-1532, (2002).

[4] I. J. G. Sparrow, D. A. Sager, C. B. E. Gawith, P. G. R. Smith, G. D. Emmerson, M. Kaczmarek, A. Dyadyusha. Quant. Electron. \& Laser Science Conference, 2, 963, (2005). 